\title{
Overview of Orientation
}

\section{Relationship Models}

\section{A.1 Nishiyama-Wassermann (NW)}

The transformation $T_{\mathrm{NW} 1}$ is uniquely defined through our unified approach (cf. Section 2) as the transformation that:

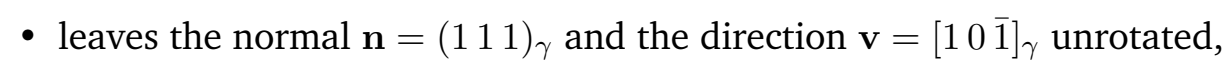

- has pure stretch component $U_{2}$.

The resulting transformation strain is

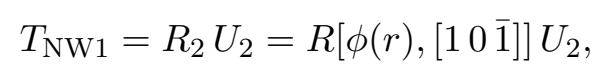

where $\phi(r)=\arccos \left(\frac{1+\sqrt{2} r}{\sqrt{3} \sqrt{1+r^{2}}}\right)$. The corresponding OR matrix is

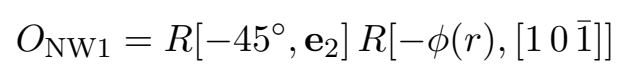

which yields the OR

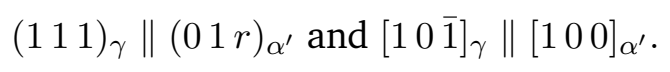

The application of $\mathcal{P}^{24}$ yields the remaining eleven NW ORs (cf. Table A1). Note that, unlike Table 1, Table A1 takes the tetragonality of the b.c.t. lattice into account 
and the b.c.t. vectors are given in a way that is consistent with the transformation strains and not only up to crystallographic equivalence.

Tab. A1. The NW orientation relationships. The corresponding transformation strain in each row is

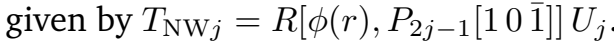

\begin{tabular}{|c|c|c|c|c|c|}
\hline $\mathrm{OR}^{\mathrm{a}}$ & f.c.c. plane $e^{b}$ & b.c.c. plane & f.c.c. direction ${ }^{\mathrm{d}}$ & b.c.c. direction ${ }^{\mathrm{e}}$ & Bain Variant $\mathrm{f}^{\mathrm{f}}$ \\
\hline NW1 & $(111)_{\gamma}$ & $(01 r)_{\alpha^{\prime}}$ & {$\left[\begin{array}{lll}1 & 0 & \overline{1}\end{array}\right]_{\gamma}$} & {$\left[\begin{array}{lll}1 & 0 & 0\end{array}\right]_{\alpha^{\prime}}$} & $U_{2}$ \\
\hline NW2 & $(111)_{\gamma}$ & $(r \quad 01)_{\alpha^{\prime}}$ & {$\left[\begin{array}{lll}1 & 1 & 0\end{array}\right]_{\gamma}$} & {$\left[\begin{array}{lll}0 & 1 & 0\end{array}\right]_{\alpha^{\prime}}$} & $U_{3}$ \\
\hline NW3 & $(111)_{\gamma}$ & $(1 r 0)_{\alpha^{\prime}}$ & {$\left[\begin{array}{lll}0 & \overline{1} & 1\end{array}\right]_{\gamma}$} & {$\left[\begin{array}{lll}0 & 0 & 1\end{array}\right]_{\alpha^{\prime}}$} & $U_{1}$ \\
\hline NW4 & $(\overline{1} 11)_{\gamma}$ & $(\bar{r} 10)_{\alpha^{\prime}}$ & {$\left[\begin{array}{lll}1 & 0 & 1\end{array}\right]_{\gamma}$} & {$\left[\begin{array}{lll}0 & 0 & 1\end{array}\right]_{\alpha^{\prime}}$} & $U_{2}$ \\
\hline NW5 & $(\overline{1} 11)_{\gamma}$ & $(0 r 1)_{\alpha^{\prime}}$ & {$\left[\begin{array}{lll}\overline{1} & \overline{1} & 0\end{array}\right]_{\gamma}$} & {$\left[\begin{array}{lll}1 & 0 & 0\end{array}\right]_{\alpha^{\prime}}$} & $U_{3}$ \\
\hline NW6 & $(\overline{1} 11)_{\gamma}$ & $(\overline{1} 0 r)_{\alpha^{\prime}}$ & {$\left[\begin{array}{lll}0 & 1 & 1\end{array}\right]_{\gamma}$} & {$\left[\begin{array}{lll}0 & 1 & 0\end{array}\right]_{\alpha^{\prime}}$} & $U_{1}$ \\
\hline NW7 & $(1 \overline{1} 1)_{\gamma}$ & $(r \overline{1} 0)_{\alpha^{\prime}}$ & {$\left[\begin{array}{lll}1 & 0 & 1\end{array}\right]_{\gamma}$} & {$\left[\begin{array}{lll}0 & 0 & 1\end{array}\right]_{\alpha^{\prime}}$} & $U_{2}$ \\
\hline NW8 & $(1 \overline{1} 1)_{\gamma}$ & $(0 \bar{r} 1)_{\alpha^{\prime}}$ & {$\left[\begin{array}{lll}1 & 1 & 0\end{array}\right]_{\gamma}$} & {$\left[\begin{array}{lll}1 & 0 & 0\end{array}\right]_{\alpha^{\prime}}$} & $U_{3}$ \\
\hline NW9 & $(1 \overline{1} 1)_{\gamma}$ & $(10 \bar{r})_{\alpha^{\prime}}$ & {$\left[\begin{array}{lll}0 & \overline{1} & 1\end{array}\right]_{\gamma}$} & {$\left[\begin{array}{lll}0 & \overline{1} & 0\end{array}\right]_{\alpha^{\prime}}$} & $U_{1}$ \\
\hline NW10 & $(11 \overline{1})_{\gamma}$ & $(r 10)_{\alpha^{\prime}}$ & {$\left[\begin{array}{lll}\overline{1} & 0 & \overline{1}\end{array}\right]_{\gamma}$} & {$\left[\begin{array}{lll}0 & 0 & \overline{1}\end{array}\right]_{\alpha^{\prime}}$} & $U_{2}$ \\
\hline NW11 & $(11 \overline{1})_{\gamma}$ & $(0 r \overline{1})_{\alpha^{\prime}}$ & {$\left[\begin{array}{lll}1 & 1 & 0\end{array}\right]_{\gamma}$} & {$\left[\begin{array}{lll}1 & 0 & 0\end{array}\right]_{\alpha^{\prime}}$} & $U_{3}$ \\
\hline NW12 & $(11 \overline{1})_{\gamma}$ & $(10 \bar{r})_{\alpha^{\prime}}$ & {$\left[\begin{array}{lll}0 & 1 & 1\end{array}\right]_{\gamma}$} & {$\left[\begin{array}{lll}0 & 1 & 0\end{array}\right]_{\alpha^{\prime}}$} & $U_{1}$ \\
\hline
\end{tabular}




\section{A.2 Kurdjumov-Sachs (KS)}

The transformation $T_{\mathrm{KS} 1}$ is uniquely defined through our unified approach (cf. Section 2) as the transformation that:

- leaves the normal $\mathbf{n}=\left(\begin{array}{lll}1 & 1 & 1\end{array}\right)_{\gamma}$ and the direction $\mathbf{v}=\left[\begin{array}{lll}1 & 0 & \overline{1}\end{array}\right]_{\gamma}$ unrotated,

- has pure stretch component $U_{3}$.

The resulting transformation strain is

$$
T_{\mathrm{KS} 1}=R[\theta(r),[1111]] R\left[\phi(r),\left[\begin{array}{lll}
1 & 1 & 0
\end{array}\right]\right] U_{3},
$$

where $\theta(r)=\arccos \left(\frac{\sqrt{3} \sqrt{r^{2}+1}+1}{2 \sqrt{r^{2}+2}}\right)$, The corresponding OR matrix is

$$
O_{\mathrm{KS} 1}=R\left[45^{\circ}, \mathbf{e}_{3}\right] R[-\phi(r),[\overline{1} 10]] R\left[-\theta(r),\left[\begin{array}{lll}
1 & 1 & 1
\end{array}\right]\right.
$$

which yields the OR

$$
(111)_{\gamma} \|(0 r 1)_{\alpha^{\prime}} \text { and }\left[\begin{array}{lll}
1 & \overline{1}
\end{array}\right]_{\gamma} \|[11 \bar{r}]_{\alpha^{\prime}}
$$

The application of $\mathcal{P}^{24}$ yields the remaining $23 \mathrm{KS}$ ORs (cf. Table A2). Note that, unlike Table 2, Table A2 takes the tetragonality of the b.c.t. lattice into account and the b.c.t. vectors are given in a way that is consistent with the transformation strains and not only up to crystallographic equivalence. 
Tab. A2. The KS orientation relationships. The corresponding transformation strain in each row is given by $T_{\mathrm{KS} j}=R\left[\theta(r), P_{j}\left[\begin{array}{lll}1 & 1 & 1\end{array}\right] R\left[\phi(r), P_{j}\left[\begin{array}{lll}1 & 1 & 0\end{array}\right] U_{j}\right.\right.$.

\begin{tabular}{|c|c|c|c|c|c|}
\hline $\mathrm{OR}^{\mathrm{a}}$ & f.c.c. plane $\mathrm{p}^{\mathrm{b}}$ & b.c.c. plane ${ }^{c}$ & f.c.c. direction ${ }^{\mathrm{d}}$ & b.c.c. direction ${ }^{\mathrm{e}}$ & Bain Variant $\mathrm{f}^{\mathrm{f}}$ \\
\hline KS1 & $(111)_{\gamma}$ & $(0 r 1)_{\alpha^{\prime}}$ & {$\left[\begin{array}{lll}1 & 0 & \overline{1}\end{array}\right]_{\gamma}$} & {$[11 \bar{r}]_{\alpha^{\prime}}$} & $U_{3}$ \\
\hline $\mathrm{KS} 2$ & $(\overline{1} \overline{1} \overline{1})_{\gamma}$ & $(\overline{1} \bar{r} 0)_{\alpha^{\prime}}$ & {$\left[\begin{array}{lll}1 & 0 & \overline{1}\end{array}\right]_{\gamma}$} & {$[r \overline{1} \overline{1}]_{\alpha^{\prime}}$} & $U_{1}$ \\
\hline KS3 & $(111)_{\gamma}$ & $(10 r)_{\alpha^{\prime}}$ & {$\left[\begin{array}{lll}1 & 1 & 0\end{array}\right]_{\gamma}$} & {$\left[\begin{array}{lll}\bar{r} & 1 & 1\end{array}\right]_{\alpha^{\prime}}$} & $U_{1}$ \\
\hline KS4 & $(\overline{1} \overline{1} \overline{1})_{\gamma}$ & $(0 \overline{1} \bar{r})_{\alpha^{\prime}}$ & {$\left[\begin{array}{lll}1 & 1 & 0\end{array}\right]_{\gamma}$} & {$[\overline{1} r \overline{1}]_{\alpha^{\prime}}$} & $U_{2}$ \\
\hline KS5 & $(111)_{\gamma}$ & $\left(\begin{array}{rll}r & 1 & 0\end{array}\right)_{\alpha^{\prime}}$ & {$\left[\begin{array}{lll}0 & 1 & 1\end{array}\right]_{\gamma}$} & {$[1 \bar{r} 1]_{\alpha^{\prime}}$} & $U_{2}$ \\
\hline KS6 & $(\overline{1} \overline{1} \overline{1})_{\gamma}$ & $(\bar{r} 0 \overline{1})_{\alpha^{\prime}}$ & {$\left[\begin{array}{lll}0 & 1 & 1\end{array}\right]_{\gamma}$} & {$[\overline{1} \overline{1} r]_{\alpha^{\prime}}$} & $U_{3}$ \\
\hline KS7 & $(\overline{1} 11)_{\gamma}$ & $(\overline{1} r 0)_{\alpha^{\prime}}$ & {$\left[\begin{array}{lll}1 & 0 & 1\end{array}\right]_{\gamma}$} & {$\left[\begin{array}{lll}r & 1 & 1\end{array}\right]_{\alpha^{\prime}}$} & $U_{1}$ \\
\hline KS8 & $(1 \overline{1} \overline{1})_{\gamma}$ & $(0 \bar{r} \overline{1})_{\alpha^{\prime}}$ & {$\left[\begin{array}{lll}1 & 0 & 1\end{array}\right]_{\gamma}$} & {$[1 \overline{1} r]_{\alpha^{\prime}}$} & $U_{3}$ \\
\hline KS9 & $(\overline{1} 11)_{\gamma}$ & $(01 r)_{\alpha^{\prime}}$ & {$\left[\begin{array}{lll}1 & 1 & 0\end{array}\right]_{\gamma}$} & {$[\overline{1} \bar{r} 1]_{\alpha^{\prime}}$} & $U_{2}$ \\
\hline KS10 & $(1 \overline{1} \overline{1})_{\gamma}$ & $(10 \bar{r})_{\alpha^{\prime}}$ & {$\left[\begin{array}{lll}\overline{1} & \overline{1} & 0\end{array}\right]_{\gamma}$} & {$[\bar{r} \overline{1} \overline{1}]_{\alpha^{\prime}}$} & $U_{1}$ \\
\hline $\mathrm{KS} 11$ & $(\overline{1} 11)_{\gamma}$ & $(\bar{r} 01)_{\alpha^{\prime}}$ & {$\left[\begin{array}{lll}0 & 1 & 1\end{array}\right]_{\gamma}$} & {$[\overline{1} 1 \bar{r}]_{\alpha^{\prime}}$} & $U_{3}$ \\
\hline KS12 & $(1 \overline{1} \overline{1})_{\gamma}$ & $(r \overline{1} 0)_{\alpha^{\prime}}$ & {$\left[\begin{array}{llll}0 & 1 & 1\end{array}\right]_{\gamma}$} & {$\left[\begin{array}{ll}1 & r\end{array}\right]_{\alpha^{\prime}}$} & $U_{2}$ \\
\hline KS13 & $(1 \overline{1} 1)_{\gamma}$ & $(1 \bar{r} 0)_{\alpha^{\prime}}$ & {$\left[\begin{array}{lll}1 & 0 & 1\end{array}\right]_{\gamma}$} & {$[\bar{r} \overline{1} 1]_{\alpha^{\prime}}$} & $U_{1}$ \\
\hline $\mathrm{KS} 14$ & $(\overline{1} 1 \overline{1})_{\gamma}$ & $(0 r \overline{1})_{\alpha^{\prime}}$ & {$\left[\begin{array}{lll}1 & 0 & 1\end{array}\right]_{\gamma}$} & 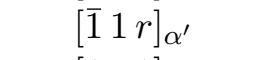 & $U_{3}$ \\
\hline $\mathrm{KS} 15$ & $(1 \overline{1} 1)_{\gamma}$ & $(0 \overline{1} r)_{\alpha^{\prime}}$ & {$\left[\begin{array}{lll}1 & 1 & 0\end{array}\right]_{\gamma}$} & {$\left[\begin{array}{lll}1 & r & 1\end{array}\right]_{\alpha^{\prime}}$} & $U_{2}$ \\
\hline KS16 & $(\overline{1} 1 \overline{1})_{\gamma}$ & $(\overline{1} 0 r)_{\alpha^{\prime}}$ & {$\left[\begin{array}{lll}1 & 1 & 0\end{array}\right]_{\gamma}$} & {$\left[\begin{array}{lll}r & 1 & \overline{1}\end{array}\right]_{\alpha^{\prime}}$} & $U_{1}$ \\
\hline $\mathrm{KS} 17$ & $(1 \overline{1} 1)_{\gamma}$ & $\left(\begin{array}{llll}r & 0 & 1\end{array}\right)_{\alpha^{\prime}}$ & {$\left[\begin{array}{lll}0 & \overline{1} & \overline{1}\end{array}\right]_{\gamma}$} & {$[1 \overline{1} \bar{r}]_{\alpha^{\prime}}$} & $U_{3}$ \\
\hline KS18 & $(\overline{1} 1 \overline{1})_{\gamma}$ & $(\bar{r} 10)_{\alpha^{\prime}}$ & {$\left[\begin{array}{lll}0 & \overline{1} & \overline{1}\end{array}\right]_{\gamma}$} & {$[\overline{1} \bar{r} \overline{1}]_{\alpha^{\prime}}$} & $U_{2}$ \\
\hline KS19 & $(11 \overline{1})_{\gamma}$ & $(1 r 0)_{\alpha^{\prime}}$ & {$\left[\begin{array}{lll}\overline{1} & 0 & \overline{1}\end{array}\right]_{\gamma}$} & {$\left[\begin{array}{lll}\bar{r} & 1 & \overline{1}\end{array}\right]_{\alpha^{\prime}}$} & $U_{1}$ \\
\hline KS20 & $(\overline{1} \overline{1} 1)_{\gamma}$ & $(0 \bar{r} 1)_{\alpha^{\prime}}$ & {$\left[\begin{array}{lll}1 & 0 & 1\end{array}\right]_{\gamma}$} & {$[\overline{1} \overline{1} \bar{r}]_{\alpha^{\prime}}$} & $U_{3}$ \\
\hline $\mathrm{KS} 21$ & $(11 \overline{1})_{\gamma}$ & $(01 \bar{r})_{\alpha^{\prime}}$ & {$\left[\begin{array}{lll}1 & \overline{1} & 0\end{array}\right]_{\gamma}$} & {$[1 \bar{r} \overline{1}]_{\alpha^{\prime}}$} & $U_{2}$ \\
\hline KS22 & $(\overline{1} \overline{1} 1)_{\gamma}$ & $(\overline{1} 0 r)_{\alpha^{\prime}}$ & {$\left[\begin{array}{lll}1 & \overline{1} & 0\end{array}\right]_{\gamma}$} & {$[r \overline{1} 1]_{\alpha^{\prime}}$} & $U_{1}$ \\
\hline KS23 & $(11 \overline{1})_{\gamma}$ & $(r 0 \overline{1})_{\alpha^{\prime}}$ & {$\left[\begin{array}{lll}0 & 1 & 1\end{array}\right]_{\gamma}$} & {$\left[\begin{array}{lll}1 & 1 & r\end{array}\right]_{\alpha^{\prime}}$} & $U_{3}$ \\
\hline KS24 & $(\overline{1} \overline{1} 1)_{\gamma}$ & $(\bar{r} \overline{1} 0)_{\alpha^{\prime}}$ & {$\left[\begin{array}{lll}0 & 1 & 1\end{array}\right]_{\gamma}$} & {$\left[\begin{array}{lll}\overline{1} & r & 1\end{array}\right]_{\alpha^{\prime}}$} & $U_{2}$ \\
\hline${ }^{\mathrm{a}} \mathrm{KS} j$ & $(111)_{\gamma}$ & $0 r 1)_{\alpha^{\prime}}$ & $P_{j}[1$ & ${ }^{\mathrm{f}} U_{j}=P_{j} U_{3} P_{j}^{\mathrm{T}}$ & \\
\hline
\end{tabular}




\section{A.3 Pitsch (PT)}

The transformation $T_{\mathrm{P} 1}$ is uniquely defined through our unified approach (cf. Section 2) as the transformation that:

- leaves the normal $\mathbf{n}=\left(\begin{array}{lll}1 & 1 & 0\end{array}\right)_{\gamma}$ and the direction $\mathbf{v}=\left[\begin{array}{lll}0 & 0 & 1\end{array}\right]_{\gamma}$ unrotated,

- has pure stretch component $U_{2}$.

The resulting transformation strain is

$$
T_{\mathrm{P} 1}=R\left[\psi(r),\left[\begin{array}{lll}
1 & 0 & 0
\end{array}\right] U_{2},\right.
$$

where $\psi(r)=-\arccos \left(\frac{\sqrt{2}+r}{\sqrt{2+r^{2}}}\right)$. The corresponding OR matrix is

$$
O_{\mathrm{P} 1}=R\left[45^{\circ}, \mathbf{e}_{2}\right] R\left[-\psi(r),\left[\begin{array}{lll}
1 & 0 & 0
\end{array}\right]\right.
$$

which yields the OR

$$
(01 \overline{1})_{\gamma} \|(\bar{r} 2 \bar{r})_{\alpha^{\prime}} \text { and }\left[\begin{array}{lll}
1 & 0 & 0
\end{array}\right]_{\gamma} \|\left[\begin{array}{lll}
1 & 0 & \overline{1}
\end{array}\right]_{\alpha^{\prime}}
$$

The application of $\mathcal{P}^{24}$ yields the remaining eleven P ORs (cf. Table A3).

\section{Remark}

$O_{\mathrm{P} 1}$ also yields the parallelism $\left[\begin{array}{lll}0 & 1 & 1\end{array}\right]_{\gamma} \|\left[\begin{array}{lll}1 & r & 1\end{array}\right]_{\alpha^{\prime}}$ stated in "The martensite transformation in thin foils of iron-nitrogen alloys" by Pitsch (for $r=1$ ). 
Tab. A3. The Pitsch orientation relationships. The corresponding transformation strain in each row is given by $T_{\mathrm{P} j}=R\left[\psi(r), P_{2 j-1}\left[\begin{array}{lll}1 & 0 & 0\end{array}\right]\right] U_{j}$.

\begin{tabular}{|c|c|c|c|c|c|}
\hline $\mathrm{OR}^{\mathrm{a}}$ & f.c.c. plane $e^{b}$ & b.c.c. plane & f.c.c. direction ${ }^{\mathrm{d}}$ & b.c.c. direction ${ }^{\mathrm{e}}$ & Bain Variant ${ }^{f}$ \\
\hline $\mathrm{P} 1$ & $(01 \overline{1})_{\gamma}$ & $(\bar{r} 2 \bar{r})_{\alpha^{\prime}}$ & {$\left[\begin{array}{lll}1 & 0 & 0\end{array}\right]_{\gamma}$} & {$\left[\begin{array}{lll}1 & 0 & \overline{1}\end{array}\right]_{\alpha^{\prime}}$} & $U_{2}$ \\
\hline $\mathrm{P} 2$ & $(\overline{1} 01)_{\gamma}$ & $(\bar{r} \bar{r} 2)_{\alpha^{\prime}}$ & {$\left[\begin{array}{lll}0 & 1 & 0\end{array}\right]_{\gamma}$} & {$\left[\begin{array}{lll}1 & 1 & 0\end{array}\right]_{\alpha^{\prime}}$} & $U_{3}$ \\
\hline P3 & $(1 \overline{1} 0)_{\gamma}$ & $(2 \bar{r} \bar{r})_{\alpha^{\prime}}$ & {$\left[\begin{array}{lll}0 & 0 & 1\end{array}\right]_{\gamma}$} & {$\left[\begin{array}{lll}0 & \overline{1} & 1\end{array}\right]_{\alpha^{\prime}}$} & $U_{1}$ \\
\hline $\mathrm{P} 4$ & $(110)_{\gamma}$ & $(r 2 \bar{r})_{\alpha^{\prime}}$ & {$\left[\begin{array}{lll}0 & 0 & 1\end{array}\right]_{\gamma}$} & {$\left[\begin{array}{lll}1 & 0 & 1\end{array}\right]_{\alpha^{\prime}}$} & $U_{2}$ \\
\hline P5 & $(0 \overline{1} 1)_{\gamma}$ & $(r \bar{r} 2)_{\alpha^{\prime}}$ & {$\left[\begin{array}{lll}1 & 0 & 0\end{array}\right]_{\gamma}$} & {$\left[\begin{array}{lll}1 & 1 & 0\end{array}\right]_{\alpha^{\prime}}$} & $U_{3}$ \\
\hline $\mathrm{P} 6$ & $(\overline{1} 0 \overline{1})_{\gamma}$ & $(\overline{2} \bar{r} \bar{r})_{\alpha^{\prime}}$ & {$\left[\begin{array}{lll}0 & 1 & 0\end{array}\right]_{\gamma}$} & {$\left[\begin{array}{lll}0 & 1 & 1\end{array}\right]_{\alpha^{\prime}}$} & $U_{1}$ \\
\hline P7 & $(\overline{1} \overline{1} 0)_{\gamma}$ & $(\bar{r} \overline{2} \bar{r})_{\alpha^{\prime}}$ & {$\left[\begin{array}{lll}0 & 0 & 1\end{array}\right]_{\gamma}$} & {$\left[\begin{array}{lll}1 & 0 & 1\end{array}\right]_{\alpha^{\prime}}$} & $U_{2}$ \\
\hline P8 & $(011)_{\gamma}$ & $(\bar{r} r 2)_{\alpha^{\prime}}$ & {$\left[\begin{array}{lll}1 & 0 & 0\end{array}\right]_{\gamma}$} & {$\left[\begin{array}{lll}1 & 1 & 0\end{array}\right]_{\alpha^{\prime}}$} & $U_{3}$ \\
\hline P9 & $(10 \overline{1})_{\gamma}$ & $(2 r \bar{r})_{\alpha^{\prime}}$ & {$\left[\begin{array}{lll}0 & 1 & 0\end{array}\right]_{\gamma}$} & {$\left[\begin{array}{lll}0 & \overline{1} & 1\end{array}\right]_{\alpha^{\prime}}$} & $U_{1}$ \\
\hline P10 & $(\overline{1} 10)_{\gamma}$ & $(\bar{r} 2 r)_{\alpha^{\prime}}$ & {$\left[\begin{array}{lll}0 & 0 & \overline{1}\end{array}\right]_{\gamma}$} & {$\left[\begin{array}{lll}\overline{1} & 0 & \overline{1}\end{array}\right]_{\alpha^{\prime}}$} & $U_{2}$ \\
\hline P11 & $(0 \overline{1} \overline{1})_{\gamma}$ & $(\bar{r} \bar{r} \overline{2})_{\alpha^{\prime}}$ & {$\left[\begin{array}{lll}1 & 0 & 0\end{array}\right]_{\gamma}$} & {$\left[\begin{array}{lll}1 & \overline{1} & 0\end{array}\right]_{\alpha^{\prime}}$} & $U_{3}$ \\
\hline $\mathrm{P} 12$ & $\left(\begin{array}{lll}1 & 0 & 1\end{array}\right)_{\gamma}$ & $(2 \bar{r} r)_{\alpha^{\prime}}$ & {$\left[\begin{array}{lll}0 & 1 & 0\end{array}\right]_{\gamma}$} & {$\left[\begin{array}{lll}0 & 1 & 1\end{array}\right]_{\alpha^{\prime}}$} & $U_{1}$ \\
\hline $\begin{array}{l}{ }^{\mathrm{a}} \mathrm{P}_{j} \\
{ }^{\mathrm{f}} U_{j}=\end{array}$ & $\begin{array}{l}P_{2 j-1}(01 \overline{1})_{\gamma} \\
j-1 U_{2} P_{2 j-1}^{\mathrm{T}}\end{array}$ & ${ }^{c} P_{2 j-1}(\bar{r} 2$ & $P_{2 j-1}[10$ & ${ }_{2 j-1}(10 \overline{1})_{c}$ & \\
\hline
\end{tabular}




\section{A.4 Greninger-Troiano (GT)}

The transformation $T_{\mathrm{GT} 1}$ is uniquely defined through our unified approach (cf. Section 2) as the transformation that:

- leaves the normal $\mathbf{n}=(111)_{\gamma}$ and the direction $\mathbf{v}=[\overline{5} 17 \overline{12}]_{\gamma}$ unrotated,

- has pure stretch component $U_{3}$.

The resulting transformation strain is

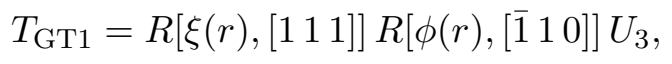

where $\xi(r)=\arccos \left(\frac{7^{2}+17^{2} \sqrt{3} \sqrt{1+r^{2}}}{\sqrt{2} \sqrt{5^{2}+12^{2}+17^{2}} \sqrt{7^{2}+17^{2}+17^{2} r^{2}}}\right)$. The corresponding OR matrix is

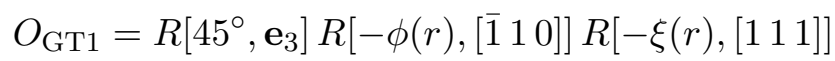

which yields the OR

$$
(111)_{\gamma} \|(0 r 1)_{\alpha^{\prime}} \text { and }[\overline{12} \overline{5} 17]_{\gamma} \|[\overline{7} \overline{17} 17 r]_{\alpha^{\prime}} .
$$

The application of $\mathcal{P}^{24}$ yields the remaining 23 GT ORs (cf. Table A4).

\section{Example}

Let $r=1.045$ (as in "The mechanism of martensite formation" by Greninger

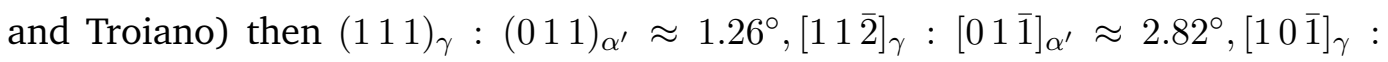
$[11 \overline{1}]_{\alpha^{\prime}} \approx 2.94^{\circ}$ and $[0 \overline{1} 1]_{\gamma}:[1 \overline{1} 1]_{\alpha^{\prime}} \approx 7.86^{\circ}$. 
Tab. A4. The GT orientation relationships. The corresponding transformation strain in each row is given by $T_{\mathrm{GT} j}=R\left[\xi(r), P_{j}\left[\begin{array}{lll}1 & 1 & 1\end{array}\right] R\left[\phi(r), P_{j}\left[\begin{array}{lll}1 & 1 & 0\end{array}\right] U_{j}\right.\right.$.

\begin{tabular}{|c|c|c|c|c|c|}
\hline $\mathrm{OR}^{\mathrm{a}}$ & f.c.c. plane $\mathrm{e}^{\mathrm{b}}$ & b.c.c. plane ${ }^{\mathrm{c}}$ & f.c.c. direction ${ }^{\mathrm{d}}$ & b.c.c. direction ${ }^{\mathrm{e}}$ & Bain Variant ${ }^{\mathrm{f}}$ \\
\hline GT1 & $(111)_{\gamma}$ & $(0 r 1)_{\alpha^{\prime}}$ & {$[\overline{12} \overline{5} 17]_{\gamma}$} & {$[\overline{7} \overline{17} 17 r]_{\alpha^{\prime}}$} & $U_{3}$ \\
\hline GT2 & $(\overline{1} \overline{1} \overline{1})_{\gamma}$ & $(\overline{1} \bar{r} 0)_{\alpha^{\prime}}$ & {$\left[\begin{array}{lll}17 & 5 & 12\end{array}\right]_{\gamma}$} & {$\left[\begin{array}{llll}17 r & 17 & 7\end{array}\right]_{\alpha^{\prime}}$} & $U_{1}$ \\
\hline GT3 & $(111)_{\gamma}$ & $(10 r)_{\alpha^{\prime}}$ & {$[17 \overline{12} \overline{5}]_{\gamma}$} & {$[17 r \overline{7} \overline{17}]_{\alpha^{\prime}}$} & $U_{1}$ \\
\hline GT4 & $(\overline{1} \overline{1} \overline{1})_{\gamma}$ & $(0 \overline{1} \bar{r})_{\alpha^{\prime}}$ & {$[12.17] 5_{\gamma}$} & {$\left[\begin{array}{lll}7 & \overline{17 r} & 17\end{array}\right]_{\alpha^{\prime}}$} & $U_{2}$ \\
\hline GT5 & $(111)_{\gamma}$ & $\left(\begin{array}{lll}r & 10\end{array}\right)_{\alpha^{\prime}}$ & {$\left[\begin{array}{lll}\overline{5} & 17 & \overline{12}\end{array}\right]_{\gamma}$} & {$[\overline{17} 17 r \overline{7}]_{\alpha^{\prime}}$} & $U_{2}$ \\
\hline GT6 & $(\overline{1} \overline{1} \overline{1})_{\gamma}$ & $(\bar{r} 0 \overline{1})_{\alpha^{\prime}}$ & {$\left[\begin{array}{lll}5 & 12 & \overline{17}\end{array}\right]_{\gamma}$} & {$[177 \overline{17 r}]_{\alpha^{\prime}}$} & $U_{3}$ \\
\hline GT7 & $(\overline{1} 11)_{\gamma}$ & $(\overline{1} r 0)_{\alpha^{\prime}}$ & {$[\overline{17} \overline{5} \overline{12}]_{\gamma}$} & {$[\overline{17 r} \overline{17} \overline{7}]_{\alpha^{\prime}}$} & $U_{1}$ \\
\hline GT8 & $(1 \overline{1} \overline{1})_{\gamma}$ & $(0 \bar{r} \overline{1})_{\alpha^{\prime}}$ & {$[\overline{12} 5 \overline{17}]_{\gamma}$} & {$[\overline{7} 17 \overline{17 r}]_{\alpha^{\prime}}$} & $U_{3}$ \\
\hline GT9 & $(\overline{1} 11)_{\gamma}$ & $(01 r)_{\alpha^{\prime}}$ & {$\left[\begin{array}{lll}12 & 17 & \overline{5}\end{array}\right]_{\gamma}$} & {$\left[\begin{array}{lll}7 & 17 r & 17\end{array}\right]_{\alpha^{\prime}}$} & $U_{2}$ \\
\hline GT10 & $(1 \overline{1} \overline{1})_{\gamma}$ & $(10 \bar{r})_{\alpha^{\prime}}$ & {$\left[\begin{array}{llll}1 & 1 & 12 & 5\end{array}\right]_{\gamma}$} & {$\left[\begin{array}{llll}17 r & 7 & 17\end{array}\right]_{\alpha^{\prime}}$} & $U_{1}$ \\
\hline GT11 & $(\overline{1} 11)_{\gamma}$ & $(\bar{r} 01)_{\alpha^{\prime}}$ & {$\left[\begin{array}{lll}\overline{5} & 12 & \overline{17}\end{array}\right]_{\gamma}$} & {$\left[\begin{array}{lll}17 & 7 & 17 r\end{array}\right]_{\alpha^{\prime}}$} & $U_{3}$ \\
\hline GT12 & $(1 \overline{1} \overline{1})_{\gamma}$ & $(r \overline{1} 0)_{\alpha^{\prime}}$ & {$\left[\begin{array}{lll}5 & 17 & \overline{12}\end{array}\right]_{\gamma}$} & {$[\overline{17} \overline{17 r} 7]_{\alpha^{\prime}}$} & $U_{2}$ \\
\hline GT13 & $(1 \overline{1} 1)_{\gamma}$ & $(1 \bar{r} 0)_{\alpha^{\prime}}$ & {$[175 \overline{12}]_{\gamma}$} & {$[17 r 17 \overline{7}]_{\alpha^{\prime}}$} & $U_{1}$ \\
\hline GT14 & $(\overline{1} 1 \overline{1})_{\gamma}$ & $(0 r \overline{1})_{\alpha^{\prime}}$ & {$\left[\begin{array}{lll}12 & \overline{5} & \overline{17}\end{array}\right]_{\gamma}$} & {$\left[\begin{array}{lll}7 & \overline{17} & \overline{17 r}\end{array}\right]_{\alpha^{\prime}}$} & $U_{3}$ \\
\hline GT15 & $(1 \overline{1} 1)_{\gamma}$ & $(0 \overline{1} r)_{\alpha^{\prime}}$ & {$[\overline{12} \overline{17} \overline{5}]_{\gamma}$} & {$\left[\begin{array}{lll}\overline{7} & \overline{17 r} & \overline{17}\end{array}\right]_{\alpha^{\prime}}$} & $U_{2}$ \\
\hline GT16 & $(\overline{1} 1 \overline{1})_{\gamma}$ & $(\overline{1} 0 r)_{\alpha^{\prime}}$ & {$[\overline{17} \overline{12} 5]_{\gamma}$} & {$[\overline{17 r} \overline{7} 17]_{\alpha^{\prime}}$} & $U_{1}$ \\
\hline GT17 & $(1 \overline{1} 1)_{\gamma}$ & 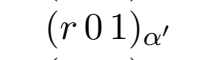 & {$\left[\begin{array}{llll}5 & 12 & 17\end{array}\right]_{\gamma}$} & {$[\overline{17} 717 r]_{\alpha^{\prime}}$} & $U_{3}$ \\
\hline GT18 & $(\overline{1} 1 \overline{1})_{\gamma}$ & $(\bar{r} 10)_{\alpha^{\prime}}$ & {$\left[\begin{array}{lll}5 & 17 & 12\end{array}\right]_{\gamma}$} & {$[1717 r 7]_{\alpha^{\prime}}$} & $U_{2}$ \\
\hline GT19 & $(11 \overline{1})_{\gamma}$ & $(1 r 0)_{\alpha^{\prime}}$ & {$[17 \overline{5} 12]_{\gamma}$} & {$[17 r \overline{17} 7]_{\alpha^{\prime}}$} & $U_{1}$ \\
\hline GT20 & $(\overline{1} \overline{1} 1)_{\gamma}$ & $(0 \bar{r} 1)_{\alpha^{\prime}}$ & {$\left[\begin{array}{lll}12 & 5 & 17\end{array}\right]_{\gamma}$} & {$\left[\begin{array}{llll}7 & 17 & 17 r\end{array}\right]_{\alpha^{\prime}}$} & $U_{3}$ \\
\hline GT21 & $(11 \overline{1})_{\gamma}$ & $(01 \bar{r})_{\alpha^{\prime}}$ & {$[\overline{12} 175]_{\gamma}$} & {$\left[\begin{array}{llll}7 & 17 & 17\end{array}\right]_{\alpha^{\prime}}$} & $U_{2}$ \\
\hline GT22 & $(\overline{1} \overline{1} 1)_{\gamma}$ & $(\overline{1} 0 r)_{\alpha^{\prime}}$ & {$[\overline{17} 12 \overline{5}]_{\gamma}$} & {$\left[\begin{array}{lll}\overline{17 r} & 7 & \overline{17}\end{array}\right]_{\alpha^{\prime}}$} & $U_{1}$ \\
\hline GT23 & $(11 \overline{1})_{\gamma}$ & $(r \quad 0 \overline{1})_{\alpha^{\prime}}$ & {$\left[\begin{array}{lll}\overline{5} & \overline{12} & \overline{17}\end{array}\right]_{\gamma}$} & {$[\overline{17} \overline{7} \overline{17 r}]_{\alpha^{\prime}}$} & $U_{3}$ \\
\hline GT24 & $(\overline{1} \overline{1} 1)_{\gamma}$ & $(\bar{r} \overline{1} 0)_{\alpha^{\prime}}$ & {$\left[\begin{array}{lll}5 & \overline{17} & \overline{12}\end{array}\right]_{\gamma}$} & {$[17 \overline{17 r} \overline{7}]_{\alpha^{\prime}}$} & $U_{2}$ \\
\hline
\end{tabular}




\section{A.5 Inverse Greninger-Troiano (GT’)}

The transformation $T_{\mathrm{GT}^{\prime} 1}$ is uniquely defined through our unified approach (cf. Section 2) as the transformation that:

- leaves the normal $\mathbf{n}=(\overline{17} \overline{7} 17)_{\gamma}$ and the direction $\mathbf{v}=\left[\begin{array}{lll}1 & 0 & 1\end{array}\right]_{\gamma}$ unrotated,

- has pure stretch component $U_{3}$.

The resulting transformation strain is

$$
T_{\mathrm{GT}^{\prime} 1}=R\left[\iota(r),\left[\begin{array}{lll}
1 & 0 & 1
\end{array}\right]\right] R\left[-\psi(r),\left[\begin{array}{lll}
0 & 1 & 0
\end{array}\right] U_{3}=R\left[\iota(r),\left[\begin{array}{lll}
1 & 0 & 1
\end{array}\right] R_{\mathrm{P} 2} U_{3}\right.\right.
$$

where $\iota(r)=\arccos \left(\frac{17^{2} \sqrt{2} \sqrt{2+r^{2}}+7^{2} r}{\sqrt{17^{2}+17^{2}+7^{2}} \sqrt{2 \cdot 17^{2}+7^{2} r^{2}+17^{2} r^{2}}}\right)$. The corresponding OR matrix is

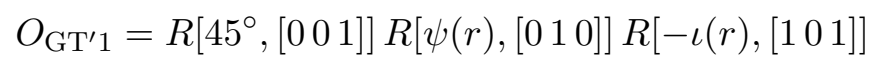

which yields the OR

$$
(\overline{17} \overline{7} 17)_{\gamma} \|(\overline{5 r} 1 \overline{2} r 17)_{\alpha^{\prime}} \text { and }\left[\begin{array}{lll}
1 & 0 & 1
\end{array}\right]_{\gamma} \|[11 r]_{\alpha^{\prime}} .
$$

The application of $\mathcal{P}^{24}$ yields the remaining $23 \mathrm{GT}^{\prime}$ ORs (cf. Table A5). 
Tab. A5. The $\mathrm{GT}^{\prime}$ orientation relationships. The corresponding transformation strain in each row is given by $T_{\mathrm{GT}^{\prime} j}=R\left[\iota(r), P_{j}\left[\begin{array}{lll}1 & 0 & 1\end{array}\right]\right] R\left[-\psi(r), P_{j}\left[\begin{array}{lll}0 & 1 & 0\end{array}\right] U_{j}\right.$.

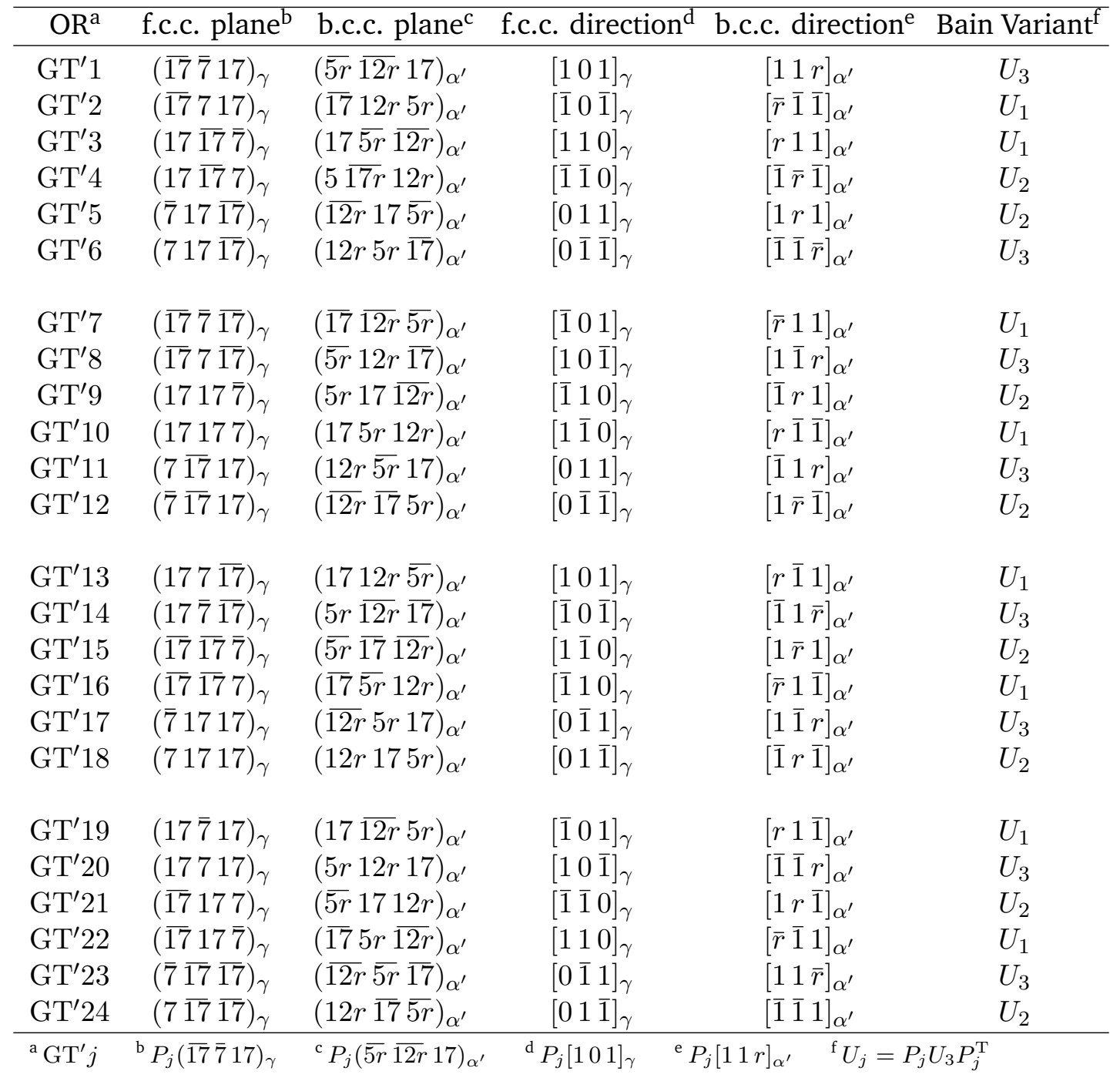




\section{The Group $\mathcal{P}^{24}$}

The elements of $\mathcal{P}^{24}$ in the standard Euclidean basis $\left\{\mathbf{e}_{1}, \mathbf{e}_{2}, \mathbf{e}_{3}\right\}$ are given by

$$
\begin{aligned}
& P_{1}=\mathbf{1}=\left(\begin{array}{lll}
1 & 0 & 0 \\
0 & 1 & 0 \\
0 & 0 & 1
\end{array}\right) \\
& P_{2}=R\left[180^{\circ}, \mathbf{e}_{1}-\mathbf{e}_{3}\right]=\left(\begin{array}{ccc}
0 & 0 & -1 \\
0 & -1 & 0 \\
-1 & 0 & 0
\end{array}\right), \\
& P_{3}=R\left[120^{\circ}, \mathbf{e}_{1}+\mathbf{e}_{2}+\mathbf{e}_{3}\right]=\left(\begin{array}{ccc}
0 & 0 & 1 \\
1 & 0 & 0 \\
0 & 1 & 0
\end{array}\right), \\
& P_{4}=R\left[180^{\circ}, \mathbf{e}_{2}-\mathbf{e}_{3}\right]=\left(\begin{array}{ccc}
-1 & 0 & 0 \\
0 & 0 & -1 \\
0 & -1 & 0
\end{array}\right), \\
& P_{5}=R\left[-120^{\circ}, \mathbf{e}_{1}+\mathbf{e}_{2}+\mathbf{e}_{3}\right]=\left(\begin{array}{ccc}
0 & 1 & 0 \\
0 & 0 & 1 \\
1 & 0 & 0
\end{array}\right), \\
& P_{6}=R\left[180^{\circ}, \mathbf{e}_{1}-\mathbf{e}_{2}\right]=\left(\begin{array}{ccc}
0 & -1 & 0 \\
-1 & 0 & 0 \\
0 & 0 & -1
\end{array}\right), \\
& P_{7}=R\left[-90^{\circ}, \mathbf{e}_{2}\right]=\left(\begin{array}{ccc}
0 & 0 & -1 \\
0 & 1 & 0 \\
1 & 0 & 0
\end{array}\right), \\
& P_{8}=R\left[180^{\circ}, \mathbf{e}_{1}\right]=\left(\begin{array}{ccc}
1 & 0 & 0 \\
0 & -1 & 0 \\
0 & 0 & -1
\end{array}\right) \\
& P_{9}=R\left[180^{\circ}, \mathbf{e}_{2}+\mathbf{e}_{3}\right]=\left(\begin{array}{ccc}
-1 & 0 & 0 \\
0 & 0 & 1 \\
0 & 1 & 0
\end{array}\right), \quad P_{10}=R\left[-120^{\circ}, \mathbf{e}_{1}-\mathbf{e}_{2}+\mathbf{e}_{3}\right]=\left(\begin{array}{ccc}
0 & 0 & 1 \\
-1 & 0 & 0 \\
0 & -1 & 0
\end{array}\right), \\
& P_{11}=R\left[90^{\circ}, \mathbf{e}_{3}\right]=\left(\begin{array}{ccc}
0 & -1 & 0 \\
1 & 0 & 0 \\
0 & 0 & 1
\end{array}\right), \quad P_{12}=R\left[120^{\circ}, \mathbf{e}_{1}+\mathbf{e}_{2}-\mathbf{e}_{3}\right]=\left(\begin{array}{ccc}
0 & 1 & 0 \\
0 & 0 & -1 \\
-1 & 0 & 0
\end{array}\right), \\
& P_{13}=R\left[180^{\circ}, \mathbf{e}_{1}+\mathbf{e}_{3}\right]=\left(\begin{array}{ccc}
0 & 0 & 1 \\
0 & -1 & 0 \\
1 & 0 & 0
\end{array}\right) \\
& P_{14}=R\left[180^{\circ}, \mathbf{e}_{2}\right]=\left(\begin{array}{ccc}
-1 & 0 & 0 \\
0 & 1 & 0 \\
0 & 0 & -1
\end{array}\right), \\
& P_{15}=R\left[90^{\circ}, \mathbf{e}_{1}\right]=\left(\begin{array}{ccc}
1 & 0 & 0 \\
0 & 0 & -1 \\
0 & 1 & 0
\end{array}\right), \quad P_{16}=R\left[-120^{\circ}, \mathbf{e}_{1}+\mathbf{e}_{2}-\mathbf{e}_{3}\right]=\left(\begin{array}{ccc}
0 & 0 & -1 \\
1 & 0 & 0 \\
0 & -1 & 0
\end{array}\right) \text {, } \\
& P_{17}=R\left[-90^{\circ}, \mathbf{e}_{3}\right]=\left(\begin{array}{ccc}
0 & 1 & 0 \\
-1 & 0 & 0 \\
0 & 0 & 1
\end{array}\right), \quad P_{18}=R\left[120^{\circ},-\mathbf{e}_{1}+\mathbf{e}_{2}+\mathbf{e}_{3}\right]=\left(\begin{array}{ccc}
0 & -1 & 0 \\
0 & 0 & 1 \\
-1 & 0 & 0
\end{array}\right), \\
& P_{19}=R\left[90^{\circ}, \mathbf{e}_{2}\right]=\left(\begin{array}{ccc}
0 & 0 & 1 \\
0 & 1 & 0 \\
-1 & 0 & 0
\end{array}\right), \quad P_{20}=R\left[180^{\circ}, \mathbf{e}_{3}\right]=\left(\begin{array}{ccc}
-1 & 0 & 0 \\
0 & -1 & 0 \\
0 & 0 & 1
\end{array}\right), \\
& P_{21}=R\left[-90^{\circ}, \mathbf{e}_{1}\right]=\left(\begin{array}{ccc}
1 & 0 & 0 \\
0 & 0 & 1 \\
0 & -1 & 0
\end{array}\right), \quad P_{22}=R\left[-120^{\circ},-\mathbf{e}_{1}+\mathbf{e}_{2}+\mathbf{e}_{3}\right]=\left(\begin{array}{ccc}
0 & 0 & -1 \\
-1 & 0 & 0 \\
0 & 1 & 0
\end{array}\right), \\
& P_{23}=R\left[180^{\circ}, \mathbf{e}_{1}+\mathbf{e}_{2}\right]=\left(\begin{array}{ccc}
0 & 1 & 0 \\
1 & 0 & 0 \\
0 & 0 & -1
\end{array}\right), \quad P_{24}=R\left[120^{\circ}, \mathbf{e}_{1}-\mathbf{e}_{2}+\mathbf{e}_{3}\right]=\left(\begin{array}{ccc}
0 & -1 & 0 \\
0 & 0 & -1 \\
1 & 0 & 0
\end{array}\right) \text {. }
\end{aligned}
$$




\section{List of Figures}

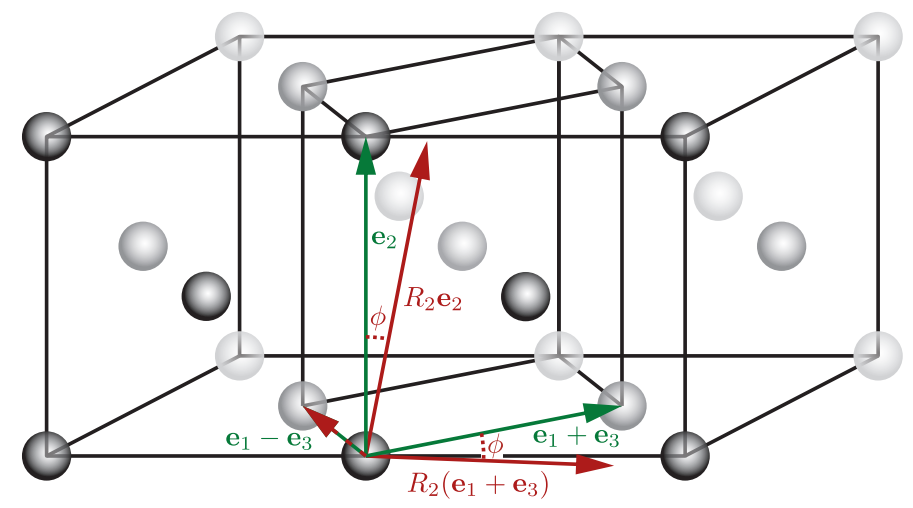

Fig. C.1. The green vectors $\mathbf{e}_{1}-\mathbf{e}_{3}, \mathbf{e}_{2}, \mathbf{e}_{1}+\mathbf{e}_{3}$ are along the edges of the tetragonal b.c.t. cell that is contained in the f.c.c. lattice and the red vectors are obtained through the rotation $R_{2}$.

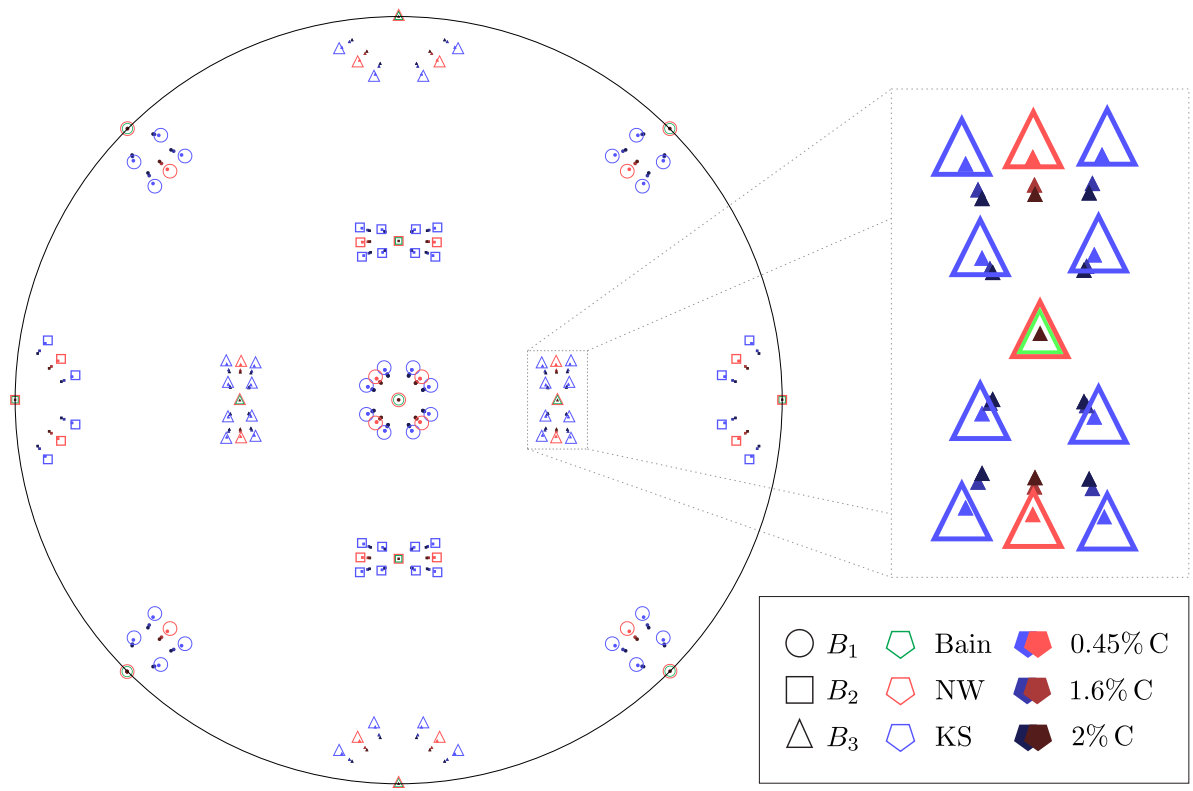

Fig. C.3. $\{100\}$ pole figures showing the change in the ORs with increasing carbon content. Hollow circles, squares and triangles correspond respectively to the f.c.c. to b.c.c. transformations with stretch components $B_{1}, B_{2}$ and $B_{3}$. The colours blue, red and green correspond respectively to KS, NW and Bain. The solid shapes correspond to increasing carbon content from lighter to darker shading and with values $0.45,1.6$ and $2 \mathrm{wt} \% \mathrm{C}$ respectively. 